\title{
FINANCIAL PERFORMANCE FORECASTING USING PROPHET MODEL: AN APPLICATION IN BIST MANUFACTURING SECTOR
}

\section{DOI: 10.17261/Pressacademia.2021.1507}

PAP- V.14-2021(33)-p.131-132

\section{Ali Yurttabir ${ }^{1}$, Ilker Kiymetli Sen ${ }^{2}$}

${ }^{1}$ Istanbul Commerce University, Institute of Social Sciences, Department of Accounting and Auditing, Istanbul, Turkey. ali.yurttabir@istanbulticaret.edu.tr, ORCID: 0000-0001-8689-3476

${ }^{2}$ Istanbul Commerce University, Faculty of Business, Department of Accounting and Auditing, Istanbul, Turkey.

ilksen@ticaret.edu.tr, ORCID: 0000-0001-6175-3397

To cite this document

Yurttabir, A., Kiymetli Sen, İ., (2021). Financial performance forecasting using prophet model: an application in BIST manufacturing sector. PressAcademia Procedia (PAP), 14, 131-132.

Permanent link to this document: http://doi.org/10.17261/Pressacademia.2021.1507

Copyright: Published by PressAcademia and limited licensed re-use rights only.

\begin{abstract}
Purpose- Through processing big data, companies have started to prefer artificial intelligence methods that can reach results faster than classical statistical methods. For this reason, financial performance forecasting studies with artificial intelligence methods have been increasing in recent years. The purpose of this study is to contribute to the gap in the field of financial performance forecasting with the Facebook Prophet model which has not been applied before although there are other time series models in the literature.

Methodology- The study employs Facebook Prophet artificial learning model by choosing the net profit/loss as target variable for financial performance forecasting on Python program with the data of 173 companies in the BIST Manufacturing Sector between 2009 and 2020, obtained from the Public Disclosure Platform.

Findings- After training the 46 periods of the data set, performance matrices of MSE, RMSE and MAPE were measured for the last 2 periods as MSE values between 0.0185 and 25.0147, RMSE values between 0.1361 and 5.0015 and MAPE values between 0.1002 and 4.6634 . In addition to values close to zero, there are also values that move away from zero. The analysis reveals that besides successful predictions, there are also unsuccessful predictions.

Conclusion- It may be concluded that the Facebook Prophet method will save companies time without requiring much effort. To further improve accuracy and performance, creating a mixed artificial intelligence model by leveraging the strengths of multiple models is recommended for further studies.
\end{abstract}

Keywords: Financial performance forecasting, artificial intelligence, time series, Facebook Prophet, BIST manufacturing sector JEL Codes: C22, C45, C53

\section{FINANSAL PERFORMANS TAHMININDE PROPHET MODELi: IMALAT SEKTÖRÜ UYGULAMASI}

\section{ÖZET}

Amaç- Büyük veriyi işleme noktasında şirketler klasik istatistik yöntemlerinden daha hızlı sonuca ulaşabilecekleri yapay zeka yöntemlerini tercih etmeye başlamışlardır. Bu nedenle son yıllarda yapay zeka yöntemleriyle finansal performans tahmini çalışmaları da giderek artmaktadır. Farklı zaman serisi modelleri ile finansal performans tahmini çalışmaları olmasına rağmen Facebook Prophet modeli ile yapılan çalışmaya literatürde rastlanmadığından alandaki boşluğa katkı sağlanması amaçlanmaktadır.

Yöntem- BIST İmalat Sektöründe yer alan 173 şirketin Kamuyu Aydınlatma Platformu veri tabanından elde edilen $2009-2020$ yılları arasındaki verileri, net dönem kar/zararı hedef değişken olarak seçilerek yapay öğrenme modeli Prophet ile Python programı üzerinde çalışarak finansal performans tahmini yapılmıştır.

Bulgular- Veri setindeki 46 dönem eğitildikten sonra son 2 döneminin tahminine ait MSE değerleri 0,0185 ile 25,0147 arasında, RMSE değerleri 0,1361 ile 5,0015 ve MAPE değerleri ise 0,1002 ile 4,6634 aralığında ölçülmüştür. Sıfıra yakın değerler yanında sıfırdan uzaklaşan değerler de bulunmaktadır. Bu da başarılı tahminlerin yanında başarısız tahminlerin de olduğunu göstermektedir.

Sonuç- Prophet yönteminin çok fazla çaba harcamayı gerektirmeden şirketlere zaman kazandıracağı söylenebilir. Doğruluğu ve performansı daha da geliştirmek için birden fazla modelin güçlü yönlerinden yararlanarak karma bir yapay zeka modeli oluşturmak sonraki çalışmalar için önerilmektedir.

Anahtar Kelimeler: Finansal performans tahmini, yapay zeka, zaman serisi, Facebook Prophet, BIST imalat sektörü. JEL Codes: C22, C45, C53 


\section{REFERENCES}

Aguilera, H., Albert, C. G., Fernández, N. N., \& Kohfahl, C. (2019). Towards flexible groundwater-level prediction for adaptive water management: using Facebook's Prophet forecasting approach. Hydrological Sciences Journal/Journal des Sciences Hydrologiques.

Akdağ, M., \& Bozma, G. (2021). Stok akış modeli ve Facebook Prophet Algoritması ile Bitcoin fiyatı tahmini. Uluslararası Ekonomi, İşletme ve Politika Dergisi, 16-30.

Aydemir, O., Ögel, S., \& Demirtaş, G. (2012). Hisse senetleri fiyatlarının belirlenmesinde finansal oranların rolü. Yönetim ve Ekonomi Dergisi, 19(2), 277-288.

Bayrakdaroğlu, A., Mirgen, C., \& Kuyu, E. (2017). Relationship between profitability ratios and stock prices: an empirical analysis on BIST-100. PressAcademia Procedia (PAP), 1-10.

Cecchetti, G., \& Kharroubi, E. (2012). Reassessing the Impact of Finance on Growth. BIS Working Papers.

Chan, W. N. (2020). Time series data mining: comparative study of ARIMA and Prophet methods for forecasting closing prices of Myanmar Stock Exchange. Journal of Computer Applications and Research, 75-80.

Duarte, D., \& Faerman, J. (2019). Comparison of time series prediction of healthcare emergency department indicators with ARIMA and Prophet. Computer Science \& Information Technology (CS \& IT) Computer Science Conference.

Gaur, S. (2020). Global forecasting of Covid-19 using ARIMA based FB-Prophet. International Journal of Engineering Applied Sciences and Technology, 463-467.

Gergin, B., \& Kıymetli Şen, İ. (2019). Kurumsal yönetim endeksinde yer almanın bankaların performansına etkisi: Borsa İstanbul'da bir araştırma. Muhasebe Bilim Dünyası Dergisi, 21(4), 956-978. doi:10.31460/mbdd.562606

Guo, C., Ge, Q., Jiang, H., Yao, G., \& Hua, Q. (2020). Maximum power demand prediction using FB-Prophet with adaptive Kalman Filtering. IEEE Access, 1-12.

Güleryüz, D., \& Özden, E. (2020). The prediction of brent crude oil trend using LSTM and Facebook Prophet. Avrupa Bilim ve Teknoloji Dergisi, $1-9$.

Hyndman, R. J., \& Athanasopoulos, G. (2018). Forecasting: Principles and Practice. Australia: OTexts.

Jha, B. K., \& Pande, S. (2021). Time series forecasting model for supermarket sales using FB-Prophet. 5th International Conference on Computing Methodologies and Communication (ICCMC). Erode,India: IEEE.

Koç, B., \& Yüncü, A. B. (2020). Muhasebe alanında 2004-2018 yılları arasında hazırlanmış lisansüstü tezlerin incelenmesi. Muhasebe Enstitüsü Dergisi(62), 63-75.

Law, S. H., \& Singh, N. (2014). Does too much finance harm economic growth? Journal of Banking and Finance, 44, 36-44.

Madhuri, C., Chinta, M., \& Kumar, V. (2020). Stock market prediction for time-series forecasting using Prophet upon ARIMA. 7th International Conference on Smart Structures and Systems (ICSSS). Chennai,India: IEEE.

Mata, A. G. (2020). A comparison between LSTM and Facebook Prophet Models: a financial forecasting case study. Universital Oberta de Catalunya.

Phutela, N., Bakshi, A., \& Gupta, S. (2020). Forecasting the stability of COVID-19 on Indian dataset with Prophet Logistic Growth Model. Research Square.

Samal, K. R., Babu, K. S., Das, S. K., \& Acharaya, A. (2019). Time series based air pollution forecasting using SARIMA and Prophet Model. ITCC 2019: Proceedings of the 2019 International Conference on Information Technology and Computer Communications, (s. 80-85).

Schumpeter, J. (1934). The Theory of Economic Development. Cambridge,MA, : Harvard University Press.

Sevli, O., \& Başer, V. G. (2020). Covid-19 salgınına yönelik zaman serisi verileri ile Prophet model kullanarak makine öğrenmesi temelli vaka tahminlemesi. Avrupa Bilim ve Teknoloji Dergisi, 827-835.

Taylor, S. J., \& Letham, B. (2017). Prophet: forecasting at scale Facebook. Eylül 29, 2021 tarihinde https://facebook.github.io/prophet/ adresinden alındı

Taylor, S. J., \& Letham, B. (2018). Forecasting at scale. The American Statistician, 72(1), 37-45. doi:10.1080/00031305.2017.1380080

Uğuz, S. (2019). Makine öğrenmesi teorik yönleri ve python uygulamaları ile bir yapay zeka ekolü. Ankara: Nobel Akademik Yayıncılık.

VeriBilimcisi.com. (2017, Temmuz 14). https://veribilimcisi.com/. Eylül 10, 2021 tarihinde Veri Bilimcisi: https://veribilimcisi.com/2017/07/14/mse-rmse-mae-mape-metrikleri-nedir/ adresinden alındı 
Weytjens, H., Lohmann, E., \& Kleinsteuber, M. (2019). Cash flow prediction: MLP and LSTM compared to ARIMA and Prophet. Springer Science+Business Media, LLC, part of Springer Nature 2019.

Žunić, E., Korjenić, K., Hodžić, K., \& Đonko, D. (2020). Applıcatıon of Facebook's Prophet Algorıthm for successful sales forecastıng based on real-world data. International Journal of Computer Science \& Information Technology, 23-36. 